\title{
CONJUGATE POINTS AND SIMPLE ZEROS FOR ORDINARY LINEAR DIFFERENTIAL EQUATIONS
}

\author{
BY \\ THOMAS L. SHERMAN( $\left.{ }^{1}\right)$
}

I. Introduction. In a very interesting paper in 1958, Hartman proved the following result [2]:

The equation

$$
L_{n} y=y^{(n)}+p_{n-1}(x) y^{(n-1)}+\cdots+p_{0}(x) y=0
$$

with $p_{i} \in C(a, b)$, has a nontrivial solution with $n$ zeros, each being counted in accordance with its multiplicity, if and only if there is a solution with $n$ distinct zeros on $(a, b)$.

Left as an open question in that paper was the question of whether the result remains true when the restriction that the interval be open is removed. The answer to this question is given in $\$ 4$ of this paper. In addition there is the problem of giving a more exact description to the solution with $n$ distinct zeros, that is, does there exist a solution with exactly $n$ distinct zeros all of which are simple? Also, can the first zero be specified? These latter two questions are answered in $\S 2$, and more specifically by

THEOREM 1. Suppose there is a solution of (1) with a zero at a and $n$ zeros on $[a, b)$. Then there is a solution with a simple zero at a whose first $n$ zeros on $[a, b)$ are simple zeros.

In $\S 3$ the results of $\S 2$ are applied to establish the continuity of the first conjugate point $\eta_{1}(t)$ of the point $t$ with respect to the coefficients in (1). In [5] it was established that $\eta_{1}(t)$ is an increasing function of $t$. The techniques used there could be used to show $\eta_{1}(t)$ is a continuous function of $t$, however an easier proof is presented here using the results of $\$ 2$ (see also [3]).

In $\$ 5$ is discussed the consequences of the existence of a solution of (1) with $n$ distinct zeros on $\left[a, \eta_{1}(a)\right]$. As is shown in $\$ 4$, such a solution need not necessarily exist.

The results of this paper are stated in terms of equation (1) but could just as easily be stated for a more general equation as in [6].

2. Existence of a solution with simple zeros. To begin with a lemma dealing with the behavior of real valued functions is proven.

Received by the editors July 19, 1968 and, in revised form, June 19, 1969.

( $\left.{ }^{1}\right)$ This work was supported by the National Science Foundation under grant GP-7398. Copyright (C) 1969, American Mathematical Society 
LEMMA 1. Let $f(x)$ and $g(x)$ be functions with the following properties:

(i) $g(x)$ has a zero of order $q \geqq 0$ at $x=a$ and $g^{(q)}(a)>0$,

(ii) $f(x)$ has a zero of order $p>q$ at $x=a$ and $f^{(p)}(a)>0$,

(iii) $f(x)$ and $g(x)$ are positive on $(a, b]$,

(iv) $f(x), g(x) \in C^{p}[a, b]$.

Then given any $\alpha>0$ there is a constant $c, 0<c<\alpha$ such that $h(x)=f(x)-c g(x)$ has a simple zero at some point $t_{c} \in(a, b)$ and $h(x) \neq 0$ for $x \in\left(t_{c}, b\right]$.

Proof. Let $\alpha>0$ be given. By (i), (ii) and (iv) there is an $\varepsilon>0$ such that $f^{(p)}(x)>0$ and $g^{(q)}(x)>0$ on $[a, a+\varepsilon]$. Let

$$
\alpha_{1}=\min \left\{\alpha, \min _{x \in[a+\varepsilon, b]} f(x) / 2 \max _{x \in[a+\varepsilon, b]} g(x)\right\} .
$$

Then for any $c \leqq \alpha_{1}, h_{c}(x)=f(x)-c g(x)>0$ for $x \in[a+\varepsilon, b]$. Let $t_{c}$ be the last zero of $h_{c}(x)$ in $[a, a+\varepsilon), 0<c \leqq \alpha_{1}$. It shall now be shown that the map $H_{c}: c \rightarrow t_{c}$ is $1-1$ and $t_{c}$ is an increasing function of $c$. In fact the latter will obviously imply the former which is itself immediate since $0<c_{1}<c_{2}$ implies by (iii) $c_{1} g(x)<c_{2} g(x)$, hence $h_{c_{2}}<h_{c_{1}}$ and hence $t_{c_{1}}<t_{c_{2}}$. Also $t_{c} \rightarrow a$ as $c \rightarrow 0$. Further, since $H_{c}$ is $1-1$, and $\left(0, \alpha_{1}\right]$ is uncountable, so is the image set of elements $t_{c}$. Hence there is an infinite number of distinct limit points $\left\{t_{c_{j}}\right\} ; t_{c_{1}}>t_{c_{2}}>\cdots$ converging to $a$ as $j \rightarrow \infty$, for which there is a set of image points $t_{c_{j i}} \rightarrow t_{c_{j}}$ as $i \rightarrow \infty$ for each $j=1,2, \ldots$.

Suppose now the lemma is not true. Then each image point $t_{c}$ corresponds to a zero of order at least two of $h_{c}$ and hence a zero of

$$
W(x)=\left|\begin{array}{ll}
f(x) & g(x) \\
f^{\prime}(x) & g^{\prime}(x)
\end{array}\right| \text { at } x=t_{c}
$$

Hence, since $W$ is continuous along with its first $p-1$ derivatives, $W\left(t_{c_{g}}\right)=0$ and by Rolle's theorem $W^{(k)}\left(t_{c,}\right)=0$ for $k<p, j=1,2, \ldots$ It is immediate that $c_{j}=f\left(t_{c_{j}}\right) / g\left(t_{c_{j}}\right)=f^{\prime}\left(t_{c_{j}}\right) / g^{\prime}\left(t_{c_{j}}\right)$. Note that $h_{c_{j}}^{\prime}\left(t_{c_{j}}\right)=0$ and $f^{\prime}\left(t_{c_{j}}\right)>0$ implies $g^{\prime}\left(t_{c_{j}}\right) \neq 0$. Suppose now that it has been shown that $c_{j}=f\left(t_{c_{j}}\right) / g\left(t_{c_{j}}\right)=\cdots=f^{(k)}\left(t_{c_{j}}\right) / g^{(k)}\left(t_{c_{j}}\right)$, and $g^{(k)}\left(t_{c_{j}}\right) \neq 0$ where $0<k<p$. Hence

Now

$$
\left|\begin{array}{ll}
f^{(r)}\left(t_{c_{j}}\right) & g^{(r)}\left(t_{c_{j}}\right) \\
f^{(s)}\left(t_{c_{j}}\right) & g^{(s)}\left(t_{c_{j}}\right)
\end{array}\right|=0, \quad 0 \leqq r<s \leqq k<p
$$

$$
W^{(k)}(x)=\left|\begin{array}{ll}
f(x) & g(x) \\
f^{(k+1)}(x) & g^{(k+1)}(x)
\end{array}\right|+(k-1)\left|\begin{array}{ll}
f^{\prime}(x) & g^{\prime}(x) \\
f^{(k)}(x) & g^{(k)}(x)
\end{array}\right|+\cdots
$$

Evaluating $W^{(k)}(x)$ at $x=t_{c,}$ this becomes

$$
W^{(k)}\left(t_{c_{j}}\right)=0=\left|\begin{array}{ll}
f\left(t_{c_{j}}\right) & g\left(t_{c_{j}}\right) \\
f^{(k+1)}\left(t_{c_{j}}\right) & g^{(k+1)}\left(t_{c_{j}}\right)
\end{array}\right|
$$


Hence $f\left(t_{c_{j}}\right) g^{(k+1)}\left(t_{c_{j}}\right)-f^{(k+1)}\left(t_{c_{j}}\right) g\left(t_{c_{j}}\right)=0$. If $g^{(k+1)}\left(t_{c_{j}}\right)=0$ then $f^{(k+1)}\left(t_{c_{j}}\right)=0$ but $k+1 \leqq p$ hence $f^{(k+1)}(x)>0$ for $x \in(a, a+\varepsilon]$, hence $g^{(k+1)}\left(t_{c_{j}}\right) \neq 0$. Thus

$$
f\left(t_{c_{j}}\right)-\frac{f^{(k+1)}\left(t_{c_{j}}\right)}{g^{(k+1)}\left(t_{c_{j}}\right)} g\left(t_{c_{j}}\right)=0 \quad \text { and } \quad c_{j}=\frac{f^{(k+1)}\left(t_{c_{j}}\right)}{g^{(k+1)}\left(t_{c_{j}}\right)} \text { for all } k<p .
$$

Hence $h_{c_{j}}$ has a zero of order $p+1$ at $t_{c_{j}}$. Now letting $j \rightarrow \infty, h_{c_{j}} \rightarrow h_{0}$ also has a zero of order $p+1$ at $x=a$ which is impossible since $h_{0}(x)=f(x)\left({ }^{2}\right)$.

REMARK. A similar result holds if the roles of $a$ and $b$ are interchanged.

A series of seven lemmas are now proven which lead up to Theorem 1. Some of the results presented as lemmas are of interest in their own right.

To avoid repetition, it shall be understood that by the term solution is meant nontrivial solution.

For completeness the following definition is included.

Definition 1. The first conjugate point $\eta_{1}(a)$ of the point $a$ is the smallest number $b>a$ for which there exists a nontrivial solution of (1) which vanishes at $a$ and has $n$ zeros, counting multiplicities in $[a, b]$.

It has been proven [5] that if there is a solution with $n$ zeros on $[a, \infty)$ then $\eta_{1}(a)$ exists.

LEMMA 2. If $n \geqq 3$ and there is a solution of (1) with $n$ zeros on $[a, b)$ then there is $a$ solution of (1) with $n$ zeros on $[a, b)$ which vanishes at a and has at least one zero in $\left(a, \eta_{1}(a)\right)$.

Proof. Suppose this were not the case. Let $\phi(x)$ be a solution of (1) with a zero of order $k_{1},\left(1 \leqq k_{1}<n\right)$, at $a$ and a zero of order $k_{2} \geqq n-k_{1}$ at $\eta_{1}(a)<b$. It is known that for some $k_{1}$ such a solution exists [5]. It can further be supposed that $\phi(x)>0$ on $\left(a, \eta_{1}(a)\right)$.

Case 1. $k_{2}=1$. Let $\Psi(x)$ be a solution of (1) with zeros at $a$ and $\left(a+\eta_{1}(a)\right) / 2$ of orders $k_{1}-1$ and 1 respectively. By supposition $\Psi$ has no other zeros on $[a, b)$. $\Psi(x)$ can be chosen positive in $\left(a,\left(a+\eta_{1}(a)\right) / 2\right)$. Hence there is a point $s_{1}, \eta_{1}(a)<s_{1}$ $<b$ at which $\Psi$ and $\phi$ have the same sign and hence so do $\phi$ and $\alpha \Psi$ for any $\alpha>0$. Also for any $\alpha>0, \alpha \Psi$ crosses $\phi$ at least once in $\left(a,\left(a+\eta_{1}(a)\right) / 2\right)$. Pick $\alpha=\phi\left(s_{1}\right) / \Psi\left(s_{1}\right)$ then $z=\phi-\alpha \Psi$ has zeros at $a$ and $s_{1}$ of orders $k_{1}-1$ and 1 and a zero in $\left(a,\left(a+\eta_{1}(a)\right) / 2\right)$. A total of $n$ zeros in $(a, b)$ and a zero in $\left(a, \eta_{1}(a)\right)$ which is a contradiction.

Case 2. $k_{2}>1$. Let $\Psi(x)$ have a zero at $\eta_{1}(a)$ of order $k_{2}-2 \geqq 0$, at $\left(a+\eta_{1}(a)\right) / 2$ of order one, and at $a$ of order $n-\left(k_{2}-2\right)-2 \leqq k_{1}$. Note that $n-\left(k_{2}-2\right)-2>0$. $\Psi$ has been assigned $n-1$ zeros, hence by assumption it has no other zeros on $[a, b)$. It can be assumed that $\alpha \phi \Psi>0$ on some interval $\left[\left(s_{1}, \eta_{1}(a)\right) \cup\left(\eta_{1}(a), s_{2}\right)\right]$ where $\left(a+\eta_{1}(a)\right) / 2<s_{1}<\eta_{1}(a)<s_{2}<b$, and $\alpha$ is any positive constant. Let

$$
\alpha=\min \left(\phi\left(s_{1}\right) / \Psi\left(s_{1}\right), \phi\left(s_{2}\right) / \Psi\left(s_{2}\right)\right)
$$

${ }^{(2)}$ The author would like to thank Grant Gustafson for the proof given here of Lemma 1. 
then $z=\phi-\alpha \Psi$ has a zero at $a$ of order $n-\left(k_{2}-2\right)-2>0$, at $\eta_{1}(a)$ of order $k_{2}-2$; a zero in $\left[s_{1}, \eta_{1}(a)\right)$ of order at least one and in $\left(\eta_{1}(a), s_{2}\right]$ a zero of order at least one. A total of at least $n$ zeros with a zero in $\left(a, \eta_{1}(a)\right)$ and a zero at $a$.

LEMMA 3. Suppose there is a solution $\phi$ of (1) with $n$ zeros on $[a, b)$, a zero at $a$, and $R-1$ distinct zeros on $\left[a, \eta_{1}(a)\right)$. Suppose also no solution of $(1)$ with a zero at a and $n$ zeros on $[a, b)$ has more than $R-1$ distinct zeros on $\left[a, \eta_{1}(a)\right)$. Then the zeros of $\phi$ in $\left(a, r_{11}(a)\right)$ are simple zeros.

Proof. Suppose $\phi$ has $R+p$ distinct zeros, $p \geqq 0$, at the points $a=t_{1}<t_{2}<\cdots$ $<t_{R+p}$ where $t_{R-1}<\eta_{1}(a) \leqq t_{R}$ and $t_{R+p}<b$, of multiplicities $m_{1}, m_{2}, \ldots, m_{R+p}$ where $\sum_{i<R+p} m_{i}<n \leqq \sum_{i \leqq R+p} m_{i}$ and $\phi$ has no other zeros on $\left[t_{1}, t_{R+p}\right]$. Suppose the lemma is not true. Then there is a $j, 1<j<R$, such that $m_{j}>1$.

Let $\Psi(x)$ be a nontrivial solution of (1) such that $\Psi$ has a zero at $t_{j}$ of order $m_{j}-2$ and $\Psi$ has a zero at $\left(t_{j-1}+t_{j}\right) / 2$. Assign $n-m_{j}$ more zeros at $t_{1}, \ldots, t_{j-1}$, $t_{j+1}, \ldots, t_{R+p}$ by assigning zeros at the $t_{i}(i \neq j, i<R+p)$ of orders $m_{i}$, then assign zeros at $t_{R+p}$ until a total of $n-1$ zeros have been assigned to $\Psi$ in $\left[t_{1}, t_{R+p}\right]$.

Since $\Psi(x)$ has at least $R-1$ distinct zeros in $\left[a, \eta_{1}(a)\right)$ including a zero at $a$ and a total of $n-1$ zeros in $[a, b), \Psi$ cannot have another distinct zero in $\left[a, \eta_{1}(a)\right.$ ) nor can it vanish at $t_{j}$ with a zero of order greater than $m_{j}-2$, hence $\phi$ and $\Psi$ have no zeros in common on $\left[a, t_{R+p}\right)$ other than those assigned above.

Let $\gamma=\left(t_{j-1}+t_{j}\right) / 2$ and let $\delta=\min \left\{t_{j+1}, \eta_{1}(a)\right\}$. Since $\phi(x)$ and $-\Psi(x)$ are both solutions and the zeros of $\phi$ and $\Psi$ at $t_{j}$ are both even or both odd it can be assumed that $\phi(x) \Psi(x)>0$ for $x \in\left(\gamma, t_{j}\right) \cup\left(t_{j}, \delta\right)$ and $\Psi(x)>0$ for $x \in\left(t_{j}, \delta\right)$ (hence $\phi(x)>0$ for $\left.x \in\left(t_{j}, \delta\right)\right)$. Now since $\gamma>t_{j-1}$ and $\phi^{\left(m_{j}-2\right)}\left(t_{j}\right)=0<\Psi^{\left(m_{j}-2\right)}\left(t_{j}\right)$, for any $\alpha>0$, $\alpha \Psi^{\left(m_{j}-2\right)}\left(t_{j}\right)>\phi^{\left(m_{j}-2\right)}\left(t_{j}\right)$ and $\alpha \Psi(\gamma)=0<|\phi(\gamma)|$. Hence for any positive $\alpha$ there is a $x_{\alpha} \in\left(\gamma, t_{j}\right)$ such that $\alpha \Psi\left(x_{\alpha}\right)=\phi\left(x_{\alpha}\right)$. Let $\alpha=\phi\left(\left(t_{j}+\delta\right) / 2\right) / \Psi\left(\left(t_{j}+\delta\right) / 2\right)>0$ and $z(x)=\phi(x)-\alpha \Psi(x)$. Then $z(x)$ is a solution of (1) with zeros at $t_{1}, t_{2}, \ldots, x_{\alpha}, t_{j}$, $\left(t_{j}+\delta\right) / 2, t_{j+1}, \ldots, t_{R+p}$ and a total of $n$ zeros in $\left[t_{1}, t_{R+p}\right]$. But $z(x)$ then has $R$ distinct zeros in $\left[t_{1}, \eta_{1}(a)\right)$, contradicting the maximality of $R$.

LEMMA 4. Suppose there is a solution of (1) with $n$ zeros on $[a, b)$, a zero at $a$, and $R-1$ distinct zeros on $\left[a, \eta_{1}(a)\right)$. Suppose also no solution of (1) with a zero at a and $n$ zeros on $[a, b)$ has more than $R-1$ distinct zeros on $\left[a, \eta_{1}(a)\right)$. Then there is $a$ solution of (1) with a simple zero at $a, R-1$ distinct zeros in $\left[a, \eta_{1}(a)\right)$, and $n$ zeros on $[a, b)$.

Proof. By Lemma 3 any solution with $n$ zeros on $[a, b)$, a zero at $a$, and $R-1$ zeros on $\left[a, \eta_{1}(a)\right)$ has only simple zeros in $\left(a, \eta_{1}(a)\right)$. Let $S=\{m \mid$ there is a nontrivial solution $\phi(x)$ of (1) with $n$ zeros on $[a, b), R-1$ zeros on $\left[a, \eta_{1}(a)\right)$ and a zero of order $m>0$ at $x=a$ \}. Let $m_{1}=\min m \in S$. Suppose, if possible $m_{1}>1$. Let $\phi(x)$ be a solution of (1) having zeros at $a=t_{1}<t_{2}<\cdots<t_{R-1}<t_{R}<\cdots<t_{R+p}$, where $t_{R-1}<\eta_{1}(a) \leqq t_{R}$ and $t_{R+p}<b$, of orders $m_{1}, m_{2}=1, \ldots, m_{R-1}=1, m_{R}, \ldots, m_{R+p}$, where $\sum_{i<R+p} m_{i}<n \leqq \sum_{i \leqq R+p} m_{i}$. Let $\Psi(x)$ be a solution of (1) having zeros at $t_{1}$, 
$\left(t_{1}+t_{2}\right) / 2, t_{2}, \ldots, t_{R-1}, t_{R}, \ldots, t_{R+p}$ of orders $m_{1}-1,1, m_{2}, \ldots, m_{R-1}, m_{R}, \ldots$, $n-\sum_{i<R+p} m_{i}-1$. $\Psi$ has been assigned $n-1$ zeros and can have no others by the maximality of $R$. There are two cases.

Case 1. $m_{R+p}-\left(n-\sum_{i<R+p} m_{i}-1\right)>0$ is even. Then there is an $\varepsilon, 0<\varepsilon<b-t_{R+p}$ such that $|\Psi(x)|>|\phi(x)|>0$ for $x \in\left[t_{R+p}-\varepsilon, t_{R+p}\right) \cup\left(t_{R+p}, t_{R+p}+\varepsilon\right]$. Since both $-\phi$ and $-\Psi$ are solutions it can be supposed that $\Psi(x) \phi(x)>0$ in this set. Let

$$
\alpha=\min \left\{\phi\left(t_{R+p}-\varepsilon\right) / \Psi\left(t_{R+p}-\varepsilon\right), \phi\left(t_{R+p}+\varepsilon\right) / \Psi\left(t_{R+p}+\varepsilon\right)\right\} .
$$

Then $\alpha \Psi(x)$ crosses $\phi(x)$ at least once in $\left[t_{R+p}-\varepsilon, t_{R+p}\right)$ and at least once in $\left(t_{R+p}, t_{R+p}+\varepsilon\right]$. Let these points of intersection be $\bar{t}_{1}$ and $\bar{t}_{2}$. Then $z(x)=\phi(x)-\alpha \Psi(x)$ is a solution of (1) with zeros at $t_{1}, t_{2}, \ldots, t_{R-1}, t_{R}, \ldots, \bar{t}_{1}, t_{R+p}, \bar{t}_{2}$ of orders $m_{1}-1, p_{2}, \ldots, p_{R-1}, p_{R}, \ldots, \bar{p}_{1}, n-\sum_{i<R+p} m_{i}-1, \bar{p}_{2}$ where $p_{i} \geqq m_{i}$ and $\bar{p}_{1} \geqq 1$, $\bar{p}_{2} \geqq 1$. $z(x)$ has a total of at least $n$ zeros in $[a, b), R-1$ distinct zeros in $\left[a, \eta_{1}(a)\right.$ ) and a zero at $a$ of order $m_{1}-1$ contradicting the minimality of $m_{1}$.

Case 2. $m_{R+p}-\left(n-\sum_{i<R+p} m_{i}-1\right)>0$ is odd. Since $-\phi$ and $-\Psi$ are solutions it can be assumed that $\phi(x)>0$ and $\Psi(x)>0$ for $x \in\left(t_{1},\left(t_{1}+t_{2}\right) / 2\right)$. Since $m_{1}-1<m_{1}$ and $\phi\left(\left(t_{1}+t_{2}\right) / 2\right)>\Psi\left(\left(t_{1}+t_{2}\right) / 2\right)=0$, it follows that, for any $\alpha>0, \alpha \Psi(x)=\phi(x)$ for some $x_{\alpha} \in\left(t_{1},\left(t_{1}+t_{2}\right) / 2\right)$. Since $m_{R+p}-\left(n-\sum_{i<R+p} m_{i}-1\right)$ is odd there is an $\varepsilon$, $0<\varepsilon<b-t_{R+p}$ such that $\phi(x) \Psi(x)>0$ for $x \in\left(t_{R+p}, t_{R+p}+\varepsilon\right]$ and $|\Psi(x)|>|\phi(x)|$ in that interval. This follows since both $\phi$ and $\Psi$ have $\sum_{i<R+p} m_{i}$ zeros in $\left[a, t_{R+p}\right)$, $\sum_{1<i<R+p} m_{i}$ zeros in common in $\left[t_{2}, t_{R+p}\right)$, both start out with the same sign, $\phi$ has one more zero at $x=a$ and $\Psi$ has a zero of order one at $x=\left(t_{1}+t_{2}\right) / 2$ where as $\phi$ does not vanish there. Let $\alpha=\phi\left(t_{R+p}+\varepsilon\right) / \Psi\left(t_{R+p}+\varepsilon\right)$ then $z=\phi-\alpha \Psi$ has zeros at $t_{1}, x_{\alpha}, t_{2}, \ldots, t_{R+p-1}, t_{R+p}, t_{R+p}+\varepsilon$ of orders at least $m_{1}-1,1, m_{2}, \ldots, m_{R+p-1}$, $n-\sum_{i<R+p} m_{i}-1,1$. A total of $n$ zeros in $[a, b)$, a zero at $a$, and $R$ distinct zeros in $\left[a, \eta_{1}(a)\right)$, contradicting the choice of $R$.

LEMMA 5. Let the following hypothesis be satisfied:

(i) There is a solution $\phi(x)$ of (1) with $n$ zeros on $[a, b)$, a simple zero at $a, R-1$ distinct zeros on $\left[a, \eta_{1}(a)\right)$, and its first $n$ zeros occur at $R+p$ distinct points.

(ii) No solution of (1) with a zero at $a$ and $n$ zeros on $[a, b)$ has more than $R-1$ distinct zeros on $\left[a, \eta_{1}(a)\right)$.

(iii) No solution of (1) with a simple zero at $a$, $n$ zeros on $[a, b)$ and $R-1$ distinct zeros on $\left[a, \eta_{1}(a)\right)$ has its first $n$ zeros occurring at more than $R+p$ points.

(iv) Let the first $n$ zeros of $\phi$ occur at points $a=t_{1}<t_{2}<\cdots<t_{R+p}$ of orders $m_{1}=1, m_{2}=1, \ldots, m_{R-1}=1, m_{R}, \ldots, m_{R+p}$ (by Lemma 3) where $\sum_{i<R+p} m_{i}<n$ $\leqq \sum_{i \leqq R+p} m_{i}$.

Then, $m_{R}=m_{R+1}=\cdots=m_{R+p-1}=1$, if $p \geqq 1$.

Proof. Suppose there is a $j, R \leqq j<R+p$ such that $m_{j}>1$. Let $j$ be the smallest such integer.

Let $\Psi(x)$ be a solution of (1) such that $\Psi(x)$ has a zero at $t_{j}$ of order $m_{j}-2$ and $\Psi(x)$ has a zero of order one at $\left(t_{1}+t_{2}\right) / 2$. Assign $n-1-\left(m_{j}-1\right)$ more zeros at 
$t_{1}, t_{2}, \ldots, t_{j-1}, t_{j+1}, \ldots, t_{R+p}$ by assigning zeros at the $t_{i}(i \neq j, i<R+p)$ orders $m_{i}$, then assign zeros at $t_{R+p}$ until a total of $n-1$ zeros have been assigned to $\Psi$ in $\left[t_{1}, t_{R+p}\right]$. This can be done since

$$
\begin{aligned}
\sum_{i \leqq R+p: i \neq j} m_{i}+m_{j}-2+1 & =\sum_{i \leqq R+p} m_{i}-1 \geqq n-1>\sum_{i<R+p} m_{i}-1 \\
& =\sum_{i<R+p ; i \neq j} m_{i}+m_{j}-2+1 .
\end{aligned}
$$

$\Psi(x)$ can have no other zeros in $[a, b)$ by the choice of $R$ (note that $j \geqq R$ ).

Since both $-\phi$ and $-\Psi$ are solutions it can be assumed that $\phi(x) \Psi(x)>0$ for $x \in\left(t_{j-1}, t_{j}\right) \cup\left(t_{j}, t_{j+1}\right)$. Since $\phi$ and $\Psi$ have the same order zero at $t=a$ and, by the minimality of $j, \Psi$ has one more zero than $\phi$ on $\left[a, t_{j}\right)$, namely the zero at $\left(t_{1}+t_{2}\right) / 2$, it follows that $\phi^{\left(m_{1}\right)}(a) \Psi^{\left(m_{1}\right)}(a)<0$. Also for any constant $\alpha>0$, there is an $\varepsilon_{\alpha}>0$ such that $\alpha|\Psi(x)|>|\phi(x)|>0$ for $x \in\left[t_{j}-\varepsilon_{\alpha}, t_{j}\right) \cup\left(t_{j}, t_{j}+\varepsilon_{\alpha}\right]$ where $t_{j-1}$ $<t_{j}-\varepsilon_{\alpha}<t_{j}<t_{j}+\varepsilon_{\alpha}<t_{j+1}$.

Let

$$
\alpha=\min \left\{\frac{\phi\left(t_{j}-\varepsilon_{1}\right)}{2 \Psi\left(t_{j}-\varepsilon_{1}\right)}, \frac{\phi\left(t_{j}+\varepsilon_{1}\right)}{2 \Psi^{(}\left(t_{j}+\varepsilon_{1}\right)},\left|\frac{\phi^{\left(m_{i}\right)}\left(t_{i}\right)}{2 \Psi^{\left(m_{i}\right)}\left(t_{i}\right)}\right| i \neq j, \frac{1}{2}\right\}
$$

and let $z_{\alpha}=\phi-\alpha \Psi$. Then $z_{\alpha}$ has a zero in $\left(t_{j}-\varepsilon_{1}, t_{j}\right)$ and a zero in $\left(t_{j}, t_{j}+\varepsilon_{1}\right)$. Further $z_{\alpha}$ has zeros at $t_{1}, t_{2}, \ldots, t_{R+p}$ and a total of $n$ zeros in $\left[t_{1}, t_{R+p}\right]$ since $\phi$ and $\Psi$ had $n-2$ zeros in common. Since $z_{\beta}=\phi-\beta \Psi$ has the same properties for $0<\beta \leqq \alpha$, it must only be established that for some $\beta$ in this range, $z_{\beta}$ has no other zeros in $\left[t_{1}, t_{R+p}\right]$.

By the way $\alpha$ was chosen, there exist $\delta_{1}>0, \delta_{2}>0, \ldots, \delta_{R+p}>0$ such that

$$
\begin{aligned}
& |\phi(x)|>\beta|\Psi(x)| \\
& \text { for } x \in\left(t_{1}, t_{1}+\delta_{1}\right] \cup\left\{\bigcup_{i=2: i \neq j}^{R+p-1}\left(\left[t_{i}-\delta_{i}, t_{i}\right) \cup\left(t_{i}, t_{i}+\delta_{i}\right]\right)\right\} \cup\left[t_{R+p}-\delta_{R+p}, t_{R+p}\right)
\end{aligned}
$$

for $0<\beta \leqq \alpha$. Also $|\phi(x)|>0$ on $\bigcup_{i=1 ; i \neq j, j-1}^{R+p-1}\left[t_{i}+\delta_{i}, t_{i+1}-\delta_{i+1}\right]=S$. Let $\beta_{1}$ be defined by

then on $S$,

$$
\beta_{1}=\min \left\{\min _{x \in S}|\phi(x)| / 2 \max _{x \in S}|\Psi(x)|, \alpha\right\}
$$

$$
\beta_{1}|\Psi(x)| \leqq\left(\min _{x \in S} \frac{|\phi(x)|}{2}\right) \frac{|\Psi(x)|}{\max _{x \in S}|\Psi(x)|} \leqq \min _{x \in S} \frac{|\phi(x)|}{2}<|\phi(x)| .
$$

Since it has already been established that

$$
\begin{aligned}
|\phi(x)|> & \beta|\Psi(x)| \\
& \text { for } x \in\left[t_{1}, t_{R+p}\right]-\left[S \cup\left\{t_{1}, \ldots, t_{R+p}\right\} \cup\left(t_{j-1}+\delta_{j-1}, t_{j}\right) \cup\left(t_{j}, t_{j+1}-\delta_{j+1}\right)\right]
\end{aligned}
$$

and $0<\beta \leqq \beta_{1}$, it must only be shown for some $\beta$ in this range, $z_{\beta}(x)=\phi(x)-\beta \Psi(x)$ has only one zero in $\left(t_{j-1}, t_{j}\right)$ and only one zero in $\left(t_{j}, t_{j+1}\right)$ and these are simple zeros. 
Suppose this were not the case. Let $t_{\beta}$ be the first zero of $z_{\beta}$ in $\left(t_{j-1}, t_{j}\right)$ and $\bar{t}_{\beta}$ be the last zero of $z_{\beta}$ in $\left(t_{j}, t_{j+1}\right) . z_{\beta}$ has a zero of order $m_{j}-2$ at $t_{j}$ hence by supposition and by Rolle's theorem $z_{\beta}^{\left(m_{j}-2\right)}, z_{\beta}^{\left(m_{j}-1\right)}, z_{\beta}^{\left(m_{j}\right)}$ all vanish in $\left(t_{\beta}, \bar{t}_{\beta}\right)$. Also $t_{\beta} \rightarrow t_{j}$ and $\bar{t}_{\beta} \rightarrow t_{j}$ as $\beta \rightarrow 0$. Thus, letting $\beta \rightarrow 0, z_{0}(x)$ has a zero of order $m_{j}+1$ at $t_{j}$ which is impossible since $z_{0}(x)=\phi(x)$. Hence the desired $\beta$ exists. But for this $\beta, z_{\beta}$ has a simple zero at $a, R-1$ distinct zeros on $\left[a, \eta_{1}(a)\right]$ and its first $n$ zeros occur at, at least, $R+p+1$ points contradicting the maximality of $p$.

Lemma 6. Let the hypotheses (i), (ii), (iii) and (iv) of Lemma 5 be satisfied. Also let

(v) $m_{R+p}$ have minimum value over all solutions of (1) satisfying (i), (ii), (iii) and (iv).

Then, if $p \geqq 1, m_{R+p}=1$.

Proof. Let $\phi(x)$ be a solution satisfying the hypothesis of the lemma. Suppose the minimum $m_{R+p}>1$. Let $\Psi(x)$ be a solution of (1) with zeros at $t_{1},\left(t_{1}+t_{2}\right) / 2$, $t_{2}, \ldots, t_{R+p-2}, t_{R+p}$ of orders $m_{1}, 1, m_{2}, \ldots, m_{R+p-2}, n-(R+p-1)-1<m_{R+p}$ (by (iv)) (remember, by Lemma $5, m_{i}=1, i=1,2, \ldots, R+p-1$ ). By the maximality of $R, \Psi$ can have no other zeros on $[a, b)$. Since $-\phi$ and $-\Psi$ are also solutions, it can be assumed $\phi(x)>0$ and $\Psi(x)>0$ on $\left(t_{R+p-1}, t_{R+p}\right)$. Hence

$$
\Psi\left(t_{R+p-1}\right)>\phi\left(t_{R+p-1}\right)=0, \quad\left|\Psi^{(n-R-p)}\left(t_{R+p}\right)\right|>\phi^{(n-R-p)}\left(t_{R+p}\right)=0 .
$$

Thus, $\alpha \Psi$ starts out greater to the left of $t_{R+p}$ than $\phi$ for any $\alpha>0$. Thus there is an $\alpha>0$ such that for any $\beta, 0<\beta \leqq \alpha, \beta \Psi\left(\left(t_{R+p-1}+t_{R+p}\right) / 2\right)<\phi\left(\left(t_{R+p-1}+t_{R+p}\right) / 2\right)$ and hence there is an $x_{\beta} \in\left(t_{R+p-1},\left(t_{R+p-1}+t_{R+p}\right) / 2\right)$ and an $\bar{x}_{\beta} \in\left(\left(t_{R+p-1}+t_{R+p}\right) / 2\right.$, $\left.t_{R+p}\right)$ such that $\phi\left(x_{\beta}\right)=\beta \Psi\left(x_{\beta}\right)$ and $\phi\left(\bar{x}_{\beta}\right)=\beta \Psi\left(\bar{x}_{\beta}\right)$. Note also $\beta_{1} \neq \beta_{2}$ implies $x_{\beta_{1}} \neq x_{\beta_{2}}$ and $\bar{x}_{\beta_{1}} \neq \bar{x}_{\beta_{2}}$. Pick $\alpha_{1}=\min \left\{\alpha,\left|\phi^{\prime}\left(t_{i}\right)\right| / 2\left|\Psi^{\prime}\left(t_{i}\right)\right|\left(i=1,2, \ldots, t_{R+p-2}\right)\right\}$.

On $\left[t_{1}, t_{2}\right], \Psi$ has one more zero than $\phi$ and on $\left[t_{2}, t_{R+p-2}\right]$ they have the same number of zeros. Hence $\phi$ and $\Psi$ have opposite signs on $\left(t_{2}, t_{R+p-2}\right)-\left\{t_{3}, \ldots\right.$, $\left.t_{R+p-3}\right\}$. Also $\phi(x) \neq \beta \Psi(x)$ for $x \in\left(t_{1}, t_{2}\right)$ since this would imply $z_{\beta}=\phi-\beta \Psi$ had $n$ zeros and $R$ distinct zeros in $\left[a, \eta_{1}(a)\right)$, contrary to the choice of $R$. It will now be shown that there is an $\alpha_{2}, \alpha_{1}>\alpha_{2}>0$, such that if $0<\beta \leqq \alpha_{2}$, then $z_{\beta}$ has exactly one zero, a simple zero, in $\left(t_{R+p-1},\left(t_{R+p-1}+t_{R+p}\right) / 2\right]$.

If this were not the case then there would exist a sequence $\beta_{i} \rightarrow 0$ as $i \rightarrow \infty$ such that if $x_{\beta_{i}}$ is the last zero of $z_{\beta_{i}}$ in $\left(t_{R+p-1},\left(t_{R+p-1}+t_{R+p}\right) / 2\right]$ then $z_{\beta_{i}}^{\prime}$ vanishes, by Rolle's theorem, in $\left(t_{R+p-1}, x_{\beta_{i}}\right]$. But since $x_{\beta_{i}} \rightarrow t_{R+p-1}$ and $z_{\beta_{i}} \rightarrow \phi$ as $i \rightarrow \infty$, this implies $\phi$ has a double zero at $t_{R+p-1}$ which is impossible by Lemma 5 . Hence $\alpha_{2}>0$ exists.

Now, by Lemma 1 , there is a $\beta, 0<\beta<\alpha_{2}$, for which $z_{\beta}$ has for its first zero $\bar{x}_{\beta}$ in $\left[t_{R+p-1}, t_{R+p}\right)$ a simple zero. Hence $z_{\beta}$ has zeros at $t_{1}, t_{2}, \ldots, t_{R+p-2}, x_{\beta}, \bar{x}_{\beta}, t_{R+p}$ of orders $m_{1}, m_{2}, \ldots, m_{R+p-2}, 1,1, n-(R+p)$, a total of $n$ zeros at, at least, $R+p$ points. If $n-(R+p)=0$ then $z_{\beta}$ has as its $R+p$ th zero a simple zero, 
contradicting the minimality of $m_{R+p}$. If $n-(R+p)>0$ then $z_{\beta}$ has $n$ zeros at, at least, $R+p+1$ points contradicting the maximality of $p$.

Lemma 7. Let the hypothesis (i), (ii), (iii), (iv), and (v) of Lemma 6 be satisfied. Assume also if $\eta_{1}\left(\left(a+\eta_{1}(a)\right) / 2\right)$ exists (note that $\eta_{1}(a)$ is an increasing function of a by [3]) then $\eta_{1}(a)<b<\eta_{1}\left(\left(a+\eta_{1}(a)\right) / 2\right)$. Then $R=n$, if $p=0$.

REMARK The last assumption of this lemma is not a significant restriction since none of the previous results depended on $b$ and $\eta_{1}(a)<b$ always holds.

Proof. Suppose this is not the case. Then $R<n$ and $m_{R} \geqq n-(R-1)>1$, and hence $m_{R}-[(n-R)-1] \geqq 2$. There are two cases.

Case 1. $m_{R}-[(n-R)-1]$ is even. Let $\Psi(x)$ be a solution of (1) with zeros at $t_{1},\left(t_{1}+t_{2}\right) / 2, t_{2}, \ldots, t_{R-1}, t_{R}$ of orders $m_{1}, 1, m_{2}, \ldots, m_{R-1}, n-R-1<m_{R}$, a total of $n-1$ zeros and $R$ distinct zeros in $\left[a, \eta_{1}(a)\right)$. Hence $\Psi$ has no other zeros on $[a, b)$. It can be assumed $\phi(x) \Psi(x)>0$ for $x \in\left[t_{R}-\varepsilon, t_{R}\right) \cup\left(t_{R}, t_{R}+\varepsilon\right]$ for some $\varepsilon>0$ chosen so that $t_{R-1}<t_{R}-\varepsilon<t_{R}<t_{R}+\varepsilon<b$. Also for each $\beta, 0<\beta$, there is an $\varepsilon_{\beta}$ such that $0<|\phi(x)|<\beta|\Psi(x)|$ for $x \in\left(t_{R}-\varepsilon_{\beta}, t_{R}\right) \cup\left(t_{R}, t_{R}+\varepsilon_{\beta}\right)$. Pick

$$
\alpha=\min \left\{\phi\left(t_{R}-\varepsilon\right) / 2 \Psi\left(t_{R}-\varepsilon\right), \phi\left(t_{R}+\varepsilon\right) / 2 \Psi\left(t_{R}+\varepsilon\right),\left|\phi^{\prime}\left(t_{i}\right)\right| / 2\left|\Psi^{\prime}\left(t_{i}\right)\right| \quad(i<R)\right\} .
$$

Then, using the same technique as used in Lemma 5 , there is a $\beta_{1}, 0<\beta_{1} \leqq \alpha$, such that for $0<\beta \leqq \beta_{1}, z_{\beta}=\phi-\beta \Psi$ has no zeros in $\left[t_{1}, t_{R}-\varepsilon\right]$ other than simple zeros at $t_{1}, \ldots, t_{R-1}$. By Lemma 1 there is a $\beta, 0<\beta \leqq \beta_{1}$ for which the first zero of $z_{\beta}$ in $\left[t_{R}-\varepsilon, t_{R}\right)$ is a simple zero. Since $z_{\beta}$ also vanishes at least once in $\left(t_{R}, t_{R}+\varepsilon\right), z_{\beta}$ has $n$ zeros on $[a, b)$ and its first $n$ zeros at, at least $R+1$ points, contradicting the assumption that $p=0$.

Case 2. $m_{R}-[(n-R)-1]$ is odd. Let $\Psi_{1}$ be a solution of (1) with zeros at $t_{1}, t_{3}, \ldots, t_{R-1}, s_{1}, t_{R}$ where $t_{R} \geqq \eta_{1}(a)>s_{1}>\max \left(\left(a+\eta_{1}(a)\right) / 2, t_{R-1}\right)$ of orders $m_{1}, m_{3}, \ldots, m_{R-1}, 1, n-R<m_{R}$, a total of $n-1$ zeros with $R-1$ distinct zeros in $\left[a, \eta_{1}(a)\right)$.

If $\Psi_{1}$ has no other zeros in $\left(a, t_{R}\right]$ then it can be assumed that $\Psi_{1}>0$ and $\phi(x)>0$ for $x \in\left(t_{1}, t_{2}\right)$. Let $\alpha=\min \left\{\left|\phi^{\prime}\left(t_{i}\right)\right| / 2\left|\Psi_{1}^{\prime \prime}\left(t_{i}\right)\right| \quad(i<R)\right\} \quad$ (if $\Psi_{1}^{\prime \prime}(a)=0$ the term $\left|\phi^{\prime}\left(t_{1}\right)\right| / 2\left|\Psi_{1}^{\prime}\left(t_{1}\right)\right|$ can be omitted). Then, since on $\left[t_{1}, t_{R-1}\right], \phi$ has one more zero than $\Psi_{1}$ and on $\left[t_{R-1}, t_{R}\right), \phi$ has one less zero than $\Psi_{1}$ for any $\beta, 0<\beta \leqq \alpha, z_{\beta}=\phi-\beta \Psi$ has a zero at some point $x_{\beta} \in\left(t_{1}, t_{2}\right)$ and a zero at some point $\bar{x}_{\beta} \in\left(s_{1}, t_{R}\right)$ in addition to simple zeros at $t_{1}, t_{3}, \ldots, t_{R-1}$ and a zero at $t_{R}$ of order $n-R$, a total of at least $n$ zeros. Further the zero at $x_{\beta}$ is simple by Lemma 3 and by Lemma $1, \beta$ can be chosen so that the zero at $\bar{x}_{\beta}$ is a simple zero and $z_{\beta}$ has no other zeros in $\left[a, \bar{x}_{\beta}\right.$ ). Thus $z_{\beta}$ has $n$ zeros, a simple zero at $a, R-1$ distinct zeros on $\left[a, \eta_{1}(a)\right.$ ), and its first $n$ zeros occur at, at least, $R+1$ distinct points contradicting $p=0$.

If $\Psi_{1}$ has another zero in $\left(a, t_{R}\right]$ it occurs in $\left[\eta_{1}(a), t_{R}\right]$. First suppose $t_{R}>\eta_{1}(a)$ and the additional zero occurs in $\left[\eta_{1}(a), t_{R}\right)$. Let the first zero of $\Psi_{1}$ in $\left(s_{1}, t_{R}\right)$ be at $\gamma_{1}, \eta_{1}(a) \leqq \gamma_{1}$. It may be assumed that $\phi \Psi>0$ on $\left(s_{1}, \gamma_{1}\right)$. Let $\gamma_{2} \in\left(s_{1}, \eta_{1}(a)\right)$ and let $\alpha=\min \left\{\left|\Psi^{\prime}\left(t_{i}\right)\right| / 2\left|\phi^{\prime}\left(t_{i}\right)\right|(i<R), \Psi\left(\gamma_{2}\right) / 2 \phi\left(\gamma_{2}\right)\right\}$. Then $z_{\beta}=\Psi-\beta \phi$ for any $\beta, 0<\beta \leqq \alpha$ 
has simple zeros at $t_{1}, t_{3}, \ldots, t_{R-1}$ and (by Lemma 3$)$ at some point $\gamma_{\beta} \in\left(s_{1}, \gamma_{2}\right)$ in addition to a zero in $\left(\gamma_{2}, \gamma_{1}\right)$ and a zero of order $n-R$ at $t_{R}$, a total of at least $n$ zeros on $\left[t_{1}, t_{R}\right]$. By (ii) $z_{\beta}$ has no other zeros on $\left[t_{1}, \gamma_{2}\right]$. By Lemma 1 , there is a $\beta_{1}, 0<\beta_{1} \leqq \alpha$, such that $z_{\beta_{1}}$ has for its first zero in $\left[\gamma_{2}, \gamma_{1}\right)$ a simple zero. Hence $z_{\beta_{1}}$ has its first $n$ zeros occurring at, at least, $R+1$ distinct points, again contradicting $p=0$.

Now suppose $t_{R}=\eta_{1}(a)$ and $\Psi_{1}$ has an additional zero at $t_{R}$. Hence $\Psi_{1}$ and $\phi$ have $n-1$ zeros in common and $R-2$ common zeros on $\left[a, \eta_{1}(a)\right)$. It can be assumed that $\phi \Psi>0$ on $\left(s_{1}, t_{R}\right)$. Let $\bar{s}_{1} \in\left(s_{1}, \eta_{1}(a)\right)$ and let $z_{1}(x)=\phi(x)$ $-\left(\phi\left(\bar{s}_{1}\right) / \Psi_{1}\left(\bar{s}_{1}\right)\right) \Psi_{1}(x)$ where $\bar{s}_{1}$ is chosen so that $\phi^{\prime}\left(t_{1}\right) / \Psi_{1}^{\prime \prime}\left(t_{1}\right) \neq \phi\left(\bar{s}_{1}\right) / \Psi_{1}\left(\bar{s}_{1}\right)$ (if $\Psi_{1}^{\prime}\left(t_{1}\right)=0$, this is of course a superficial requirement). Note, this ratio is not constant on $\left(s_{1}, \eta_{1}(a)\right)$ since $\phi$ and $\Psi_{1}$ are linearly independent by the way which $\Psi_{1}$ was chosen. $z_{1}(x)$ has $n$ zeros on $\left[a, t_{R}\right]$, a simple zero at $a$, and $R-2$ zeros on $\left(a, \eta_{1}(a)\right)$ which by Lemma 3 are simple zeros. Repeat the above procedure, starting with case 1 , with $z_{1}$ taking the place of $\phi$ and $\Psi_{1}$ being replaced by $\Psi_{2}$, a solution with zeros at $t_{1}, t_{4}, \ldots, t_{R-1}, \bar{s}_{1}, s_{2}, t_{R}$, where $\eta_{1}(a)>s_{2}>\bar{s}_{1}$, of orders $m_{1}, m_{4}, \ldots$, $m_{R-1}, 1,1, n-R<m_{R}$. After at most $R-2$ steps we have a $z_{R-2}$, with zeros at $t_{1}, \bar{s}_{1}, \bar{s}_{2}, \ldots, \bar{s}_{R-2}, t_{R}$ of orders $m_{1}, 1,1, \ldots, 1, n-R+1$, which cannot have another zero at $t_{R}$ since $\left(a+\eta_{1}(a)\right) / 2<\bar{s}_{1}<\bar{s}_{2}<\cdots<\bar{s}_{R-2}<t_{R}<\eta_{1}\left(a+\eta_{1}(a)\right) / 2$. Hence the zero of $z_{R-2}$ at $t_{R}$ is of order exactly $n-R+1$. However $m_{R}-[(n-R)-1]$ $\geqq 2$ and is odd hence $m_{R}-[(n-R)-1] \geqq 3$ thus $m_{R}-[n-R+1] \geqq 1$ contradicting the minimality of $m_{R}$.

Lemma 8. Let the hypothesis of Lemma 7 be satisfied. Then if $p=0, m_{R}=1$.

Proof. From Lemma 7, $R=n$. Let $\phi(x)$ be a solution of (1) with zeros at $a=t_{1}<t_{2}<\cdots<t_{R}$ of orders $m_{1}=1, \ldots, m_{R-1}=1, m_{R}$. Suppose $m_{R}>1$. Since $\eta_{1}(a)$ is an increasing function of $a, \eta_{1}\left(t_{2}\right)>\eta_{1}(a)$, hence $\phi\left(\eta_{1}(a)\right) \neq 0$ and $t_{R}>\eta_{1}(a)$. Let $\Psi_{1}(x)$ be a solution of (1) with zeros at $t_{1}, t_{3}, \ldots, t_{n-1}, s_{1}$, where $\eta_{1}(a)>s_{1}$ $>\max \left(\left(a+\eta_{1}(a)\right) / 2, t_{n-1}\right)$ of orders $m_{1}=1, m_{3}=1, \ldots, m_{n-1}=1,1$, a total of $n-1=R-1$ zeros, all distinct on $\left[a, \eta_{1}(a)\right)$. Clearly $\Psi_{1}$ has no more zeros in $\left[a, \eta_{1}(a)\right)$. Since $\eta_{1}(a)$ is an increasing function of $a$, if $\Psi_{1}\left(\eta_{1}(a)\right)=0$ it is a simple zero and the proof is complete. Similarly if $\Psi_{1}$ vanishes in $\left(\eta_{1}(a), b\right)$ and its first zero in that interval is simple, the proof is completed. Hence either $\Psi_{1}$ vanishes in $\left(\eta_{1}(a), b\right)$ with a multiple zero as its first zero in that interval or $\Psi_{1}$ does not vanish in $\left(\eta_{1}(a), b\right)$.

Suppose first $\Psi_{1}^{\prime}$ does not vanish in $\left(\eta_{1}(a), b\right)$. It can be assumed $\phi(x) \Psi_{1}(x)>0$ on $\left(t_{1}, t_{2}\right) \cup\left(s_{1}, t_{n}\right)$. Then since $\left|\Psi_{1}\left(t_{n}\right)\right|>\left|\phi\left(t_{n}\right)\right|=0$ and $\left|\phi\left(s_{1}\right)\right|>\left|\Psi_{1}\left(s_{1}\right)\right|=0$, for any $\alpha>0, \alpha \Psi_{1}(x)=\phi(x)$ for some $x_{\alpha} \in\left(s_{1}, t_{n}\right)$. Further if $\alpha$ is chosen so that $\alpha<\phi^{\prime}\left(t_{1}\right) / \Psi_{1}^{\prime}\left(t_{1}\right)$ (note $\Psi_{1}^{\prime \prime}\left(t_{1}\right) \neq 0$ ) then $\alpha \Psi_{1}(x)=\phi(x)$ for some $\bar{x}_{\alpha} \in\left(t_{1}, t_{2}\right)$. This follows since for $\alpha$ in this range $|\phi|$ starts out larger to the right of $t_{1}$ than $\left|\Psi_{1}\right|$, but $|\phi|$ vanishes (at the point $t_{2}$ ) before $\left|\Psi_{1}\right|$. Then $z_{\alpha}(x)=\phi(x)-\alpha \Psi_{1}(x)$ has zeros at $t_{1}, \bar{x}_{\alpha}, t_{3}, \ldots, t_{n-1}$, a total of $n-1$ zeros in $\left[a, \eta_{1}(a)\right]$. Hence $z_{\alpha}$ has no other zeros 
in $\left[a, \eta_{1}(a)\right]$. Clearly $z_{\beta}, 0<\beta \leqq \alpha$ has the same properties. Lemma 1 can now be applied to show there is a $\beta$ in this range for which $z_{\beta}$ has as its first zero in $\left[\eta_{1}(a), t_{n}\right)$ a simple zero, completing the proof.

Suppose now $\Psi_{1}$ does vanish in $\left(\eta_{1}(a), b\right)$. Repeat the above proof of this lemma with $\phi$ replaced by $\Psi_{1}$, and $\Psi_{1}^{*}$ replaced by $\Psi_{2}$, a solution with zeros at $t_{1}, t_{4}, \ldots$, $s_{1}, s_{2}$, where $s_{1}<s_{2}<\eta_{1}(a)$. After at most $n-2$ repetitions a solution $\Psi_{n-2}$ is found with zeros at $t_{1}, s_{1}, s_{2}, \ldots, s_{n-2}$ where $t_{1}<\left(a+\eta_{1}(a)\right) / 2<s_{1}<s_{2}<\cdots<s_{n-2}<\eta_{1}(a)$. $\Psi_{n-2}$ cannot have a multiple zero in $\left(\eta_{1}(a), b\right)$ since if it did $\Psi_{n-2}$ would have $n$ zeros in $\left(\left(a+\eta_{1}(a)\right) / 2, \eta_{1}\left(\left(a+\eta_{1}(a)\right) / 2\right)\right)$ which is impossible. Hence repeating the proof for this solution leads to the desired conclusion.

THEOREM 1. Suppose there is a solution of (1) with a zero at a and $n$ zeros on $[a, b)$. Then there is a solution with a simple zero at a whose first $n$ zeros on $[a, b)$ are simple zeros.

Proof. If $\eta_{1}\left(\left(a+\eta_{1}(a)\right) / 2\right)$ exists let $b_{1} \in\left(\eta_{1}(a), b\right) \cap\left(\eta_{1}(a), \eta_{1}\left(\left(a+\eta_{1}(a)\right) / 2\right)\right)$. The remainder of the proof consists of reading the statements of Lemmas 2, 3, 4, $5,6,7$, and 8 with $b$ replaced by $b_{1}$.

3. Continuity of $\eta_{1}(a)$. Using Theorem 1 , it shall be shown that $\eta_{1}(a)$ is a continuous function of $a$ and the coefficients in (1). First a lemma which follows from the results of [5] will be established.

LEMMA 9. Let $a$ be any point for which $\eta_{1}(a)$ exists; then for any $b>\eta_{1}(a)$, there is a $c>a$ such that $\eta_{1}(c)=b$.

Proof. This follows from Theorem 6 of [5] after the change of variables $t=-x$.

THEOREM 2. $\eta_{1}(a)$ is a continuous function of $a$.

Proof. Let $\varepsilon>0$. By Theorem 1 there is a solution $\phi$ of (1) with a simple zero at $a$ whose first $n$ zeros on $\left[a, \eta_{1}(a)+\varepsilon\right)$ are simple zeros. Let these first $n$ zeros be at $a_{1}=t_{1}<t_{2}<\cdots<t_{n}\left(<\eta_{1}(a)+\varepsilon\right)$. Let $t_{n+1}=\min \left\{\eta_{1}(a)+\varepsilon / 2\right.$, min $b>t_{n}$ such that $\phi(b)=0\}$. Since a constant times a solution is again a solution it can be supposed that $\varepsilon<\min \left\{\max _{x \in\left[t_{i}, t_{i+1}\right]}|\phi(x)|,(i=1,2, \ldots, n)\right\}$. Since solutions are continuous functions of these initial conditions there is a $\delta_{1}>0$ such that if $a_{1} \in\left(a-\delta_{1}, a+\delta_{1}\right)$ and $\Psi$ is a solution of (1) satisfying $\Psi^{(i)}\left(a_{1}\right)=\phi^{(i)} a, i=0,1, \ldots, n-1$, then $|\phi(x)-\Psi(x)|$ $<\varepsilon$ for $x \in\left[a-\varepsilon, \eta_{1}(a)+\varepsilon\right]$ (see $[4$, p. 56]). It follows that $\Psi$ must vanish in each of the intervals $\left(t_{i}-\varepsilon, t_{i}+\varepsilon\right), i=1,2, \ldots, n$. Hence $\Psi$ has $n$ zeros on $\left[a_{1}, \eta_{1}(a)+\varepsilon\right)$ and hence $\eta_{1}\left(a_{1}\right)<\eta_{1}(a)+\varepsilon$. If $a_{1}>a$ then since $\eta_{1}(a)$ is an increasing function of $a, \eta_{1}\left(a_{1}\right) \in\left(\eta_{1}(a), \eta_{1}(a)+\varepsilon\right)$. If $a_{1}<a$ then $\eta_{1}\left(a_{1}\right)<\eta_{1}(a)$. If $\eta_{1}\left(a_{1}\right)>\eta_{1}(a)-\varepsilon$ pick $\delta=\left|a-a_{1}\right|$. If $\eta_{1}\left(a_{1}\right) \leqq \eta_{1}(a)-\varepsilon$ then by Lemma $9, \eta_{1}(a)-\varepsilon / 2=\eta_{1}(c)$ for some $c \in\left(a_{1}, a\right)$ in which case let $\delta=|a-c|<\left|a-a_{1}\right|$. Hence for any $x$ such that $|x-a|<\delta,\left|\eta_{1}(a)-\eta_{1}(x)\right|<\varepsilon$, which completes the proof (for another proof of this result see [3]). 
THEOREM 3. $\eta_{1}(a)$ is a continuous function of the coefficients $p_{i}(x), i=0,1, \ldots, n-1$.

Proof. Let $\varepsilon>0$. Let $\phi$ be a solution of (1) with a simple zero at $a$ whose first $n$ zeros on $\left[a, \eta_{1}(a)+\varepsilon\right)$ are simple zeros. Let these first $n$ zeros of $\phi$ be at $a=t_{1}<t_{2}$ $<\cdots<t_{n}$. Let $t_{n+1}=\min \left\{\eta_{1}(a)+\varepsilon / 2, \min b>t_{n}\right.$ such that $\left.\phi(b)=0\right\}$. Since a constant times a solution is again a solution it can be supposed that

$$
\varepsilon<\min \left\{\max _{x \in\left[t_{i}, t_{i}+1\right]}|\phi(x)| \quad(i=1,2, \ldots, n)\right\} .
$$

Since solutions are continuous functions of their coefficients, there is a $\delta>0$ such that if $\left|q_{i}(x)-p_{i}(x)\right|<\delta$ for $x \in\left[a, \eta_{1}(a)+\varepsilon\right]$ and $i=0,1, \ldots, n-1$, and $y_{1}(x)$ is a solution of $(1)$ and $z_{1}(x)$ is a solution of

$$
z^{(n)}+\sum_{i=0}^{n-1} q_{i}(x) z^{(i)}=0
$$

satisfying $y_{1}^{(i)}(a)=z_{1}^{(i)}(a), i=0,1, \ldots, n-1$, then $\left|y_{1}(x)-z_{1}(x)\right|<\varepsilon$ for $x \in\left[a, \eta_{1}(a)+\varepsilon\right]$. Let $\Psi(x)$ be the solution of (2) satisfying $\Psi^{(i)}(a)=\phi^{(i)}(a), i=0,1, \ldots, n-1$. Then $\Psi(x)$ has $n$ zeros on $\left[a, \eta_{1}(a)+\varepsilon\right)$. Hence if $\bar{\eta}_{1}(a)$ denotes the first conjugate point of $a$ relative to (2), $a<\bar{\eta}_{1}(a)<\eta_{1}(a)+\varepsilon$. To complete the proof it needs merely to be shown that $\bar{\eta}_{1}(a)>\eta_{1}(a)-\varepsilon$.

Suppose this were not the case. Then $\bar{\eta}_{1}(a) \leqq \eta_{1}(a)-\varepsilon$. Then, by Theorem 1 again, there is a solution $\Psi_{1}(x)$ of (2) with a simple zero at $a$ whose first $n$ zeros on $\left[a, \bar{\eta}_{1}(a)+\varepsilon / 2\right)$ are simple zeros. Let these zeros be at $a=\bar{t}_{1}<\bar{t}_{2}<\cdots<\bar{t}_{n}$. Let $\bar{t}_{n+1}=\min \left\{\eta_{1}(a)+\varepsilon / 2, \min b>\bar{t}_{n}\right.$ such that $\left.\Psi_{1}(b)=0\right\}$. Again it may be supposed that $\varepsilon<\min \left\{\max _{x \in\left[i_{i}, i_{i+1}\right]}\left|\Psi_{1}(x)\right|(i=1,2, \ldots, n)\right\}$. Hence, by the continuity of solutions with respect to the coefficients, the solution $\phi_{1}(x)$ of $(1)$ satisfying the initial conditions $\phi^{(i)}(a)=\Psi_{1}^{(i)}(a)$ has $n$ zeros on $\left[a, \bar{\eta}_{1}(a)+\varepsilon / 2\right]$. This, however, is impossible since $\bar{\eta}_{1}(a)+\varepsilon / 2<\eta_{1}(a)$. The proof of the theorem is thus complete.

4. Open, closed and half open intervals. It shall be shown by example that the interval $[a, b)$ in Theorem 1 cannot in general be replaced by the closed interval $[a, b]$.

In fact, consider the equation $y^{m}+y=0 . \eta_{1}(0)$ for this equation is achieved by a solution $\phi$ with a double zero at 0 and a simple zero at $\eta_{1}(0)$. Further it follows from Theorem 2.5 of [1] that no nontrivial solution with a double zero at $\eta_{1}(0)$ can vanish in $\left[0, \eta_{1}(0)\right)$. If there were a solution $\Psi$ with three distinct zeros on $\left[0, \eta_{1}(0)\right]$ then it would have to vanish at 0 and have a simple zero at $\eta_{1}(0)$. Hence there would exist a solution which is a linear combination of $\phi$ and $\Psi$ with a zero at 0 and a double zero at $\eta_{1}(0)$, which is impossible. A further discussion of equations of this type can be found in [1]. The existence of the solution in this example is an application of

THEOREM 4. Suppose there is a solution of (1) with $n$ zeros on $\left[a, \eta_{1}(a)\right]$, and at least $\alpha$ zeros in $\left[a, \eta_{1}(a)\right),\left(\left(a, \eta_{1}(a)\right]\right)$. Then there is a solution of $(1)$ with a zero of order at least $\alpha$ at $a\left(\eta_{1}(a)\right)$ and $n$ zeros on $\left[a, \eta_{1}(a)\right]$. 
Proof. Let $s_{1}=\left\{\phi \mid \phi\right.$ is a solution of (1) with $n$ zeros on $\left[a, \eta_{1}(a)\right]$ and at least $\alpha$ zeros in $\left.\left[a, \eta_{1}(a)\right)\right\}$. Let $s_{2}=\left\{k \mid\right.$ there is a $\phi \in s_{1}$ with a zero of order $k$ at $\left.x=a\right\}$. Let $m=\max k \in s_{2}$. Let $s_{3}=\left\{k \mid\right.$ there is a $\phi \in s_{1}$ with zero of order $m$ at $a$ and $k$ zeros in $\left.\left(a, \eta_{1}(a)\right)\right\}$. Let $M=\max k \in s_{3}$. Let $\phi$ be a solution of (1) with zeros at $a=t_{1}<t_{2}<$ $\cdots<t_{q}=\eta_{1}(a)$ of orders $m=m_{1}, m_{2}, \ldots, m_{q}$ respectively where $\alpha \leqq \sum_{i<q} m_{i}$ $\leqq n-1<\sum_{i \leqq q} m_{i}$ and $M=\sum_{1<i<q} m_{i}$.

Suppose first that $m_{1}<\alpha$. Then $q>2$ since $\phi \in s_{1}$. Let $\Psi$ be a solution of (1) with zeros at $a=t_{1}, t_{2}, \ldots, t_{q}=\eta_{1}(a)$ of orders $m_{1}+1, m_{2}, \ldots, m_{q-1}-1, n-\sum_{i<q} m_{i}-1$, a total of $n-1$ zeros in $\left[a, \eta_{1}(a)\right]$ and a total of $\sum_{i<q} m_{i} \geqq \alpha$ zeros in $\left[a, \eta_{1}(a)\right)$. By the maximality of $m_{1}, \Psi$ can have no other zeros in $\left[a, \eta_{1}(a)\right]$. By Theorem 1 of [3] there is a linear combination $\Psi_{1}$ of $\Psi$ and $\phi$ with a double zero at some point $u_{1} \in\left(t_{q-1}, t_{q}\right)$. Hence $\Psi_{1}$ has zeros at $a=t_{1}, \ldots, t_{q-1}, u_{1}, t_{q}$ of orders $m_{1}, \ldots$, $m_{q-1}-1,2, n-\sum_{i<q} m_{i}-1$. Thus $\Psi_{1} \in s_{1}$ with a zero at $a$ of order $m_{1}$, and a total of $M+1$ zeros in $\left(a, \eta_{1}(a)\right)$, contradicting the maximality of $M$. Hence $m_{1} \geqq \alpha$.

The connection between open, closed and half open intervals with regard to Theorem 1 is shown in the following theorem.

THEOREM 5. The following statements are equivalent:

(a) There is a solution of (1) with $n$ zeros on $(a, b)$.

(b) There is a solution of (1) with $n$ zeros on $[a, b)$.

(c) There is a largest point $a_{1} \in(a, b)$ such that for any $c \in\left[a, a_{1}\right), \eta_{1}(c)<b$ and for this point $a_{1}, \eta_{1}\left(a_{1}\right) \notin(a, b)$.

(d) There is a largest point $a_{2} \in(a, b)$ such that for any $c \in\left[a, a_{2}\right)$ there is a solution of (1) with a simple zero at $c$ whose first $n$ zeros on $[c, b)$ are simple zeros.

Proof. (a) $\Rightarrow$ (b) is trivial. (b) $\Rightarrow$ (c) follows since $\eta_{1}(a)$ is a continuously increasing function of $a$ (Theorem 2 above and Theorem 7 of [5]). (c) $\Rightarrow$ (d) follows from Theorem 1. (d) $\Rightarrow$ (a) is trivial.

5. Solutions with $n$ simple zeros on $\left[a, \eta_{1}(a)\right]$. The results in this section give a more complete picture of the situation when there is a solution with $n$ distinct zeros on $\left[a, \eta_{1}(a)\right]$. It is trivial to show that if there is a solution with $n$ distinct zeros on $\left[a, \eta_{1}(a)\right]$ then these zeros are simple zeros.

THEOREM 6. Suppose there is a solution $\phi_{1}(x)$ of $(1)$ with $n$ simple zeros on $\left[a, \eta_{1}(a)\right]$. Then there exist solutions $\phi_{2}, \phi_{3}, \ldots, \phi_{n-1}$ such that $\phi_{k}$ has $n$ zeros on $\left[a, \eta_{1}(a)\right], a$ zero at a of order $k, n-k-1$ simple zeros in $\left(a, \eta_{1}(a)\right)$ and no other zeros in $\left.\dot{[} a, \eta_{1}(a)\right)$. Further the zeros of $\phi_{k+1}(x)$ in $\left(a, \eta_{1}(a)\right)$ coincide with the first $n-k-2$ zeros of $\phi_{k}(x)$ in $(a, b)$.

Proof. Let the zeros of $\phi_{1}$ be at $a=t_{1}<t_{2}<\cdots<t_{n}=\eta_{1}(a)$ all of order one. Let $\phi_{2}$ be a solution of (1) with a zero at $t_{1}$ of order 2 , and simple zeros at $t_{2}, t_{3}, \ldots, t_{n-2}$, a total of $n-1$ zeros. Hence $\phi_{2}$ has no other zeros on $\left[a, \eta_{1}(a)\right)$. If $\phi_{2}$ did not vanish at $\eta_{1}(a)$ then, by Theorem 1 of [5], there is a linear combination of $\phi_{1}$ and $\phi_{2}$ with 
a double zero in $\left(t_{n-1}, t_{n}\right)$ and simple zeros at $t_{1}, \ldots, t_{n-2}$, a total of $n$ zeros in $\left[a, \eta_{1}(a)\right)$ which is impossible. Hence $\phi_{2}$ vanishes at $\eta_{1}(a)$. Suppose the existence of $\phi_{k}$ has been established then define $\phi_{k+1}$ to have a zero at $a$ of order $k+1$, and have simple zeros coincide with the first $n-k-2$ simple zeros of $\phi_{k}$ in $\left(a, \eta_{1}(a)\right)$, a total of $n-1$ zeros. Again $\phi_{k+1}$ can have no other zeros in $\left[a, \eta_{1}(a)\right)$ and if $\phi_{k+1}$ did not vanish at $\eta_{1}(a)$ there would be a linear combination of $\phi_{k}$ and $\phi_{k+1}$ with $n$ zeros in $\left[a, \eta_{1}(a)\right)$, which is impossible. Hence the existence of $\phi_{k+1}$ is established and the theorem follows by induction.

COROLLARY. Under the hypothesis of Theorem 6 there exist solutions $\phi_{2}, \phi_{3}, \ldots$, $\phi_{n-1}$ such that $\phi_{k}$ has $n$ zeros on $\left[a, \eta_{1}(a)\right]$, a zero at $\eta_{1}(a)$ of order $k, n-k-1$ simple zeros in $\left(a, \eta_{1}(a)\right)$ and no other zeros in $\left(a, \eta_{1}(a)\right]$.

LEMMA 10. Suppose there is a solution $\phi_{1}(x)$ of (1) with $n$ simple zeros on $\left[a, \eta_{1}(a)\right]$. Let $\phi_{2}, \phi_{3}, \ldots, \phi_{n-1}$ be the solutions obtained in Theorem 6 . Let the zeros of $\phi_{1}, \ldots, \phi_{n-1}$ at $\eta_{1}(a)$ be of orders $n_{1}=1, n_{2}, \ldots, n_{n-1}$. Then $n_{1} \leqq n_{2} \leqq \cdots \leqq n_{n-1}$.

Proof. Suppose this were not the case. Then there is an index $j$ such that $n_{j}>n_{j+1}$. Letting $t_{1}, \ldots, t_{n}$ be as in the proof of Theorem 6 it follows that $\phi_{j}\left(t_{n-j}\right)=0$ and $\phi_{j+1}(x) \neq 0$ for $t_{n-j} \leqq x<t_{n}$. Thus, by Theorem 1 of [5], there is a linear combination of $\phi_{j}$ and $\phi_{j+1}$ with a double zero in $\left(t_{n-j}, t_{n}\right)$, simple zeros at $t_{2}, \ldots$, $t_{n-j-1}$, and a zero at $a$ of order $j$. A total of $2+n-j-2+j=n$ zeros in $\left[a, \eta_{1}(a)\right.$ ) which is a contradiction to the definition of $\eta_{1}(a)$.

THEOREM 7. Suppose there is a solution of (1) with $n$ simple zeros on $\left[a, \eta_{1}(a)\right]$. Then there exists solutions $\Psi_{1}, \Psi_{2}, \ldots, \Psi_{n-1}$, not necessarily distinct, such that $\Psi_{k}$ has a zero at a of order at least $n-k$ and a zero at $\eta_{1}(a)$ of order at least $k$.

Proof. Let $\phi_{1}, \phi_{2}, \ldots, \phi_{n-1}$ be the solution obtained in Theorem 6. Define $\Psi_{k j}(x)(k=1,2, \ldots, n-1 ; j=k, k+1, \ldots, n-1)$ by

$$
\begin{aligned}
\Psi_{11}(x) & =\phi_{n-1}(x) ; \\
\Psi_{1 j}(x) & =\phi_{n-j}^{\prime}\left(\eta_{1}(a)\right) \phi_{n-j+1}(x)-\phi_{n-j+1}^{\prime}\left(\eta_{1}(a)\right) \phi_{n-j}(x), \quad \text { if } \phi_{n-j+1}^{\prime}\left(\eta_{1}(a)\right) \neq 0, \\
& =\phi_{n-j+1}(x), \quad \text { if } \phi_{n-j+1}^{\prime}\left(\eta_{1}(a)\right)=0 ; \\
\Psi_{k k}(x) & =\Psi_{k-1 k}(x) ; \\
\Psi_{k j}(x) & =\Psi_{k-1 j}^{(k)}\left(\eta_{1}(a)\right) \Psi_{k-1 j-1}(x)-\Psi_{k-1 j-1}^{(k)}\left(\eta_{1}(a)\right) \Psi_{k-1 j}(x), \\
& \text { if } \Psi_{k-1 j}^{(k)}\left(\eta_{1}(a)\right) \neq 0 \neq \Psi_{k-1 j-1}^{(k)}\left(\eta_{1}(a)\right), \\
& =\Psi_{k-1 j}(x), \quad \text { if } \Psi_{k-1 j}^{(k)}\left(\eta_{1}(a)\right)=0 \neq \Psi_{k-1 j-1}^{(k)}\left(\eta_{1}(a)\right), \\
& =\Psi_{k-1 j-1}(x), \quad \text { if } \Psi_{k-1 j-1}^{(k)}\left(\eta_{1}(a)\right)=0 .
\end{aligned}
$$

The claim is now that $\Psi_{k}=\Psi_{k k}$. To establish this some properties of the $\Psi_{k j}$ will first be established.

(i) $\Psi_{k j}$ has a zero at $\eta_{1}(a)$ of order at least $k+1$ for $j>k$ and of order at least $k$ if $j=k$, 
(ii) $\Psi_{k j}$ has a zero at $a$ of order at least $n-j$.

These properties will be established by induction on $k$.

First let $k=1$. For $j=1$ it is obvious. For $j>1$ the result is also obvious if $\phi_{n-j+1}^{\prime}\left(\eta_{1}(a)\right)=0$. If $\phi_{n-j+1}^{\prime}\left(\eta_{1}(a)\right) \neq 0$ then, by Lemma $9, \phi_{n-j}^{\prime}\left(\eta_{1}(a)\right) \neq 0$ and hence $\Psi_{1 j}^{\prime}\left(\eta_{1}(a)\right)=0$ and $\Psi_{1 j}$ has a zero of the same order as $\phi_{n-j}$ at $a$, namely $n-j$. This establishes (i) and (ii) for $k=1$.

Now suppose these properties hold for $k=p$. Then for $k=p+1$ and $j=p+1$, $\Psi_{p+1 p+1}(x)=\Psi_{p p+1}(x)$ hence $\Psi_{p+1 p+1}(x)$ has a zero at $\eta_{1}(a)$ of order at least $p+1$ and a zero at $a$ of order at least $n-(p+1)$. Hence $\Psi_{p+1} p+1$ has the desired properties. Suppose $k=p+1$ and $j>p+1$. If $\Psi_{p j-1}^{(p+1)}\left(\eta_{1}(a)\right)=0$ then $\Psi_{p j-1}$ has a zero at $\eta_{1}(a)$ of order at least $p+2$ and a zero at $a$ of order at least $n-(j-1)>n-j$. Hence the properties hold in this case. If $\Psi_{p j}^{(p+1)}\left(\eta_{1}(a)\right)=0 \neq \Psi_{p j-1}^{(p+1)}\left(\eta_{1}(a)\right)$ then $\Psi_{p j-1}$ has a zero at $\eta_{1}(a)$ of order at least $p+2$ and a zero at $a$ of order at least $n-j$. Hence the properties hold in this case also. If $\Psi_{p j}^{(p+1)}\left(\eta_{1}(a)\right) \neq 0 \neq \Psi_{p j-1}^{(p+1)}\left(\eta_{1}(a)\right)$ then $\Psi_{p+1}$ obviously has a zero at $\eta_{1}(a)$ of order at least $p+2$ since $\Psi_{p j}$ and $\Psi_{p j-1}$ already have zeros at $\eta_{1}(a)$ of order $p+1$. Also $\Psi_{p+1}$, has a zero at $a$ of order at least $n-j$ since both $\Psi_{p j-1}$ and $\Psi_{p j}$ have this many zeros at $a$. This (i) and (ii) hold in all cases.

These properties give the desired result.

By way of partial converse to the results, the following theorem will be established:

THEOREM 8. Suppose there are $n-1$ linearly independent solutions of $(1), \Psi_{1}, \ldots$, $\Psi_{n-1}$ such that $\Psi_{k}$ has a zero at a of order exactly $n-k$, a zero at $\eta_{1}(a)$ of order exactly $k$ and $\Psi_{k}$ does not vanish in $\left(a, \eta_{1}(a)\right)$. Then there are $n-1$ solutions $\phi_{1}, \ldots$, $\phi_{n-1}$ such that $\phi_{k}$ has a zero at a of order exactly $[(n-k+2) / 2]$, a zero at $\eta_{1}(a)$ of order exactly $[(n-k+1) / 2]$ and exactly $k-1$ distinct zeros in $\left(a, \eta_{1}(a)\right)$, where $[x]$ denotes the largest integer not exceeding $x$. In particular there is a solution, namely $\phi_{n-1}$, with $n$ simple zeros on $\left[a, \eta_{1}(a)\right]$.

Proof. It will first be established by induction that a sequence $\phi_{1}, \ldots, \phi_{n-1}$ of solutions exists such that $\phi_{k}$ has a zero at $a$ of order exactly $[(n-k+2) / 2]$, a zero at $\eta_{1}(a)$ of order exactly $[(n-k+1) / 2]$, at least $k-1$ distinct zeros in $\left(a, \eta_{1}(a)\right)$ and $\phi_{k+1}$ has at least one more distinct zero in $\left(a, \eta_{1}(a)\right)$ than $\phi_{k}$. The proof will be by induction on $k$.

If $n$ is even, then $[(n-1+2) / 2]=n / 2$ and $[(n-1+1) / 2]=n / 2$, hence $\phi_{1}$ is the solution $\Psi_{n / 2}$ which exists by hypothesis. If $n$ is odd, then $[(n-1+2) / 2]=(n+1) / 2$ and $[(n-1+1) / 2]=(n-1) / 2$, hence $\phi_{1}$ is the solution $\Psi_{(n+1) / 2}$. Suppose the result is now established for $k=p$.

Suppose first $[(n-p+2) / 2]=(n-p+1) / 2$. Then $\phi_{p}$ has a zero at $a$ of order $(n-p+1) / 2$ and a zero at $\eta_{1}(a)$ of order $(n-p+1) / 2$ and $q-1 \geqq p-1$ distinct zeros in $\left(a, \eta_{1}(a)\right)$. Let the zeros of $\phi_{p}$ be at $a=t_{0}<t_{1}<\cdots<t_{q}=\eta_{1}(a)$. Then on $\left(t_{j}, t_{j+1}\right), j=0, \ldots, q-1,\left|\phi_{p}\right|>0$. Let $m_{j}=\max _{x \in\left[t_{j}, t_{j+1}\right]}\left|\phi_{p}(x)\right|, 0 \leqq j \leqq q-1$, and let $m=\min _{0 \leqq j \leqq q-1} m_{j} . \phi_{p}$ has the same sign on at least $[(q+1) / 2]$ of the subintervals 
$\left(t_{j}, t_{j+1}\right), j=0, \ldots, q-1$. Since $-\phi_{p}$ is also a solution it may be assumed that $\phi_{p}$ is positive on at least $[(q+1) / 2]$ of these subintervals. If $q$ is even and $\phi_{p}$ is positive on exactly $q / 2$ of the subintervals, take $\phi_{p}>0$ on $\left(t_{q-1}, t_{q}\right)$. It can also be assumed that $\Psi_{(n-p-1) / 2}$ is positive on $\left(a, \eta_{1}(a)\right)$. Let $M=\max _{x \in\left[a, \eta_{1}(a)\right]} \Psi_{(n-p-1) / 2}(x)$. Then $(m / M) \Psi_{(n-p-1) / 2}$ intersects $\phi_{p}$ at least $2[(q+1) / 2] \geqq q$ distinct times on $\left(a, \eta_{1}(a)\right)$. Hence the function $\phi_{p}-(m / M) \Psi_{(n-p-1) / 2}=\phi_{p+1}$ has at least $q \geqq p$ distinct zeros on $\left(a, \eta_{1}(a)\right)$, a zero at $a$ of order

$$
\min \left(\left[\frac{n-p+2}{2}\right], n-\frac{n-p-1}{2}\right)=\frac{n-p+1}{2}=\left[\frac{n-(p+1)+2}{2}\right],
$$

and a zero at $\eta_{1}(a)$ of order

$$
\min \left(\left[\frac{n-p+1}{2}\right], \frac{n-p-1}{2}\right)=\frac{n-p-1}{2}=\left[\frac{n-(p+1)+1}{2}\right] .
$$

Suppose now $[(n-p+2) / 2]=(n-p+2) / 2$. Then $\phi_{p}$ has a zero at $a$ of order $(n-p+2) / 2$, a zero at $\eta_{1}(a)$ of order $(n-p) / 2$ and at least $q-1 \geqq p-1$ distinct zeros in $\left(a, \eta_{1}(a)\right)$. Let the $t_{p}(0 \leqq j \leqq q-1)$ and the $m$ be as chosen above. $\phi_{p}$ can be assumed positive on at least $[(q+1) / 2]$ of the subintervals $\left(t_{j}, t_{j+1}\right), j=0, \ldots, q-1$. If $q$ is even and $\phi_{p}$ is positive on exactly $q / 2$ of the subintervals, take $\phi_{p}>0$ on $\left(t_{0}, t_{1}\right) . \Psi_{(n+p) / 2}$ can be assumed positive on $\left(a, \eta_{1}(a)\right)$. Let $M=\max _{x \in\left[a, n_{1}(a)\right]} \Psi_{(n+p) / 2}$. Then as in the previous case $\phi_{p+1}=\phi_{p}-(m / M) \Psi_{(n+p) / 2}$.

To complete the proof of the theorem it need only be shown that $q>p$ is impossible. If $q>p$ then since the number of zeros of $\phi_{k}$ in $\left(a, \eta_{1}(a)\right)$ is a strictly increasing function of $k$, this would imply $\phi_{n-1}$ has at least $n-1$ distinct zeros on $\left(a, \eta_{1}(a)\right)$ and hence $n$ zeros on $\left[a, \eta_{1}(a)\right)$ which is impossible and the proof is complete.

\section{BIBLIOGRAPHY}

1. M. Hanan, Oscillation criteria for third-order linear differential equations, Pacific J. Math. 11 (1961), 919-944.

2. P. Hartman, Unrestricted n-parameter families, Rend. Circ. Mat. Palermo 7 (1958), 123-142.

3. D. B. Hinton, Disconjugate properties of a system of differential equations, J. Differential Equations 2 (1966), 420-437.

4. I. G. Petrovski, Ordinary differential equations, Prentice-Hall, New York, 1966.

5. T. Sherman, Properties of solutions of Nth order linear differential equations, Pacific J. Math. 15 (1965), 1045-1060.

6. - Properties of solutions of quasi-differential equations, Duke Math. J. 32 (1965), 297-304.

Arizona State University,

TEMPE, ARIZONA 